\title{
Luminescence dynamics in type-II GaAs/AIAs superlattices near the type-I to type-II crossover
}

\author{
Langbein, Wolfgang Werner; Kalt, H.; Hvam, Jørn Märcher
}

Published in:

Physical Review B

Link to article, DOI:

10.1103/PhysRevB.54.14589

Publication date:

1996

Document Version

Publisher's PDF, also known as Version of record

Link back to DTU Orbit

Citation $(A P A)$ :

Langbein, W. W., Kalt, H., \& Hvam, J. M. (1996). Luminescence dynamics in type-II GaAs/AlAs superlattices near the type-I to type-II crossover. Physical Review B, 54(20), 14589-14594.

https://doi.org/10.1103/PhysRevB.54.14589

\section{General rights}

Copyright and moral rights for the publications made accessible in the public portal are retained by the authors and/or other copyright owners and it is a condition of accessing publications that users recognise and abide by the legal requirements associated with these rights.

- Users may download and print one copy of any publication from the public portal for the purpose of private study or research.

- You may not further distribute the material or use it for any profit-making activity or commercial gain

- You may freely distribute the URL identifying the publication in the public portal 


\title{
Luminescence dynamics in type-II GaAs/AIAs superlattices near the type-I to type-II crossover
}

\author{
W. Langbein* and H. Kalt \\ Institut für Angewandte Physik, Universität Karlsruhe, D-76128 Karlsruhe, Germany
}

J. M. Hvam

Mikroelektronik Centret, The Technical University of Denmark, Building 345 East, DK-2800 Lyngby, Denmark

(Received 14 March 1996; revised manuscript received 27 June 1996)

\begin{abstract}
We report on a study of the time-resolved luminescence of type-II GaAs/AlAs superlattices near the type-I to type-II crossover. In spite of the slight type-II band alignment, the luminescence is dominated by the type-I transition. This is due to the inhomogeneous broadening of the type-I transition and the weak type-II oscillator strength, leading to a dominant luminescence of deeply localized type-I states. The relaxation within these localized states is found to be in agreement with a hopping model. At higher temperatures and excitation densities, the AlAs $X$ minima act as an electron reservoir, leading to a strong increase of the luminescence decay time. [S1050-2947(96)03743-2]
\end{abstract}

\section{INTRODUCTION}

The transformation of short-period GaAs/AlAs superlattices from type-I to type-II has attracted much research since it was demonstrated by Danan et al. ${ }^{1}$ This is due to the different properties of the two types of transitions in their densities of states, radiative decay times, and inhomogeneous broadenings. Subpicosecond scattering of the $\Gamma$ electrons in GaAs to the $X$ point in the adjacent AlAs layers makes these structures suitable as saturable absorbers. ${ }^{2}$ The interplay of the indirect conduction band minima $X_{x, y, z}$ was investigated by high pressure spectroscopy, ${ }^{3,4}$ revealing different strain and quantization shifts of the $X_{x, y}$ and $X_{z}$ subbands. The mixing of the $X_{z}$ and $\Gamma$ subbands due to the superlattice structure in the [001] growth direction was investigated both experimentally $^{5-7}$ and theoretically., ${ }^{8,9}$ The interface roughness of the structures plays an important role for the indirect exciton mobility ${ }^{10-14}$ and for the mixing of the $X_{x, y}$ subbands with the $\Gamma$ subband in the [001] growth direction ${ }^{12}$ and all $X$ subbands for growth directions other than $\langle 100\rangle$.

We will report, in this paper, on the influence of the inhomogeneous broadening of the type-I and type-II transition energies on the carrier energy relaxation and recombination dynamics in type-II samples near the type-II to type-I crossover. The inhomogeneous broadening is caused by the interface roughness ${ }^{15-17}$ present in the structures. In a type-I sample, photoexcited electrons relax to the $\Gamma 1$ subband minimum and recombine via the fast direct type-I recombination to the first heavy-hole valence subband (hh1). In type-II samples, electrons relax to the $X_{z} 1$ subband minimum and recombine via the weak, indirect type-II transition to the hh1. In samples near the type-I to type-II crossover, the interface fluctuations are able to swap the energy ordering of the $X_{z} 1$ and $\Gamma 1$ locally. Hence, the localized states of the $\left(X_{z} 1-\mathrm{hh} 1\right)$ excitons and those of the $(\Gamma 1-\mathrm{hh} 1)$ excitons are within the same energy range. For the $\left(X_{z} 1-\mathrm{hh} 1\right)$ excitons in samples near the crossover, the hopping to localized ( $\Gamma 1-\mathrm{hh} 1)$ states is faster than their slow radiative recombination. The photoluminescence (PL) is thus dominated by the fast recombination of localized ( $\Gamma 1-\mathrm{hh} 1)$ excitons.

\section{EXPERIMENTAL DETAILS}

We have investigated a series of GaAs/AlAs superlattices grown by molecular beam epitaxy (MBE) on (001) GaAs substrates, all designed to be near the type-II to type-I transition. We show here the results for a $2.6 \mathrm{~nm} \mathrm{GaAs}$ and 1.4 $\mathrm{nm}$ AlAs superlattice with 180 periods. The PL excitation (PLE) experiments were done using a tungsten halogen lamp dispersed by a $20 \mathrm{~cm}$ monochromator as the excitation source and a $64 \mathrm{~cm}$ spectrometer with an intensified diode array for detection, giving a resolution of $2 \mathrm{meV}$. In the low excitation time-resolved PL experiments, the samples were excited by a DCM dye laser synchronously pumped by a mode-locked

$\mathrm{Ar}^{+}$laser, emitting pulses of $10 \mathrm{ps}$ duration at an $80 \mathrm{MHz}$ repetition rate. The PL was dispersed by a $60 \mathrm{~cm}$ spectrometer and detected by a synchroscan streak camera with a time resolution of $30 \mathrm{ps}$. In the high excitation time-resolved PL experiments, the samples were excited by an excimerpumped quenched dye laser providing pulses of $70 \mathrm{ps}$ duration at $2.46 \mathrm{eV}$ with $100 \mu \mathrm{J}$ per pulse and a repetition rate of $10 \mathrm{~Hz}$. The PL was dispersed by a $27 \mathrm{~cm}$ spectrometer and temporally resolved with a single-shot streak camera.

\section{SAMPLE CHARACTERIZATION AND ORDERING OF THE TYPE-I AND TYPE-II TRANSITION}

To characterize the sample under investigation, we show in Fig. 1 the PL and the PLE spectra at a lattice temperature of $5 \mathrm{~K}$ on a logarithmic scale. The PL consists of one broad band around $1.84 \mathrm{eV}$ and a side band around $1.80 \mathrm{eV}$. The PLE shows a steplike onset due to the absorption of the ( $\Gamma 1-h h 1)$ transition with a low-energy tail. The ( $\Gamma 1-1 h 1)$ onset is only weakly visible at $1.95 \mathrm{eV}$. The excitonic features at the band edges are not pronounced in this sample. This is due to the large miniband widths of the $\Gamma 1$ subband (24 $\mathrm{meV})$ and the $\mathrm{lh} 1$ subband $(48 \mathrm{meV})$, which reduce the excitonic binding energy. ${ }^{18-20}$ The mentioned miniband widths were determined by a Kronig-Penney-type calculation. Additionally, the large inhomogeneous broadening smooths out the excitonic features. 


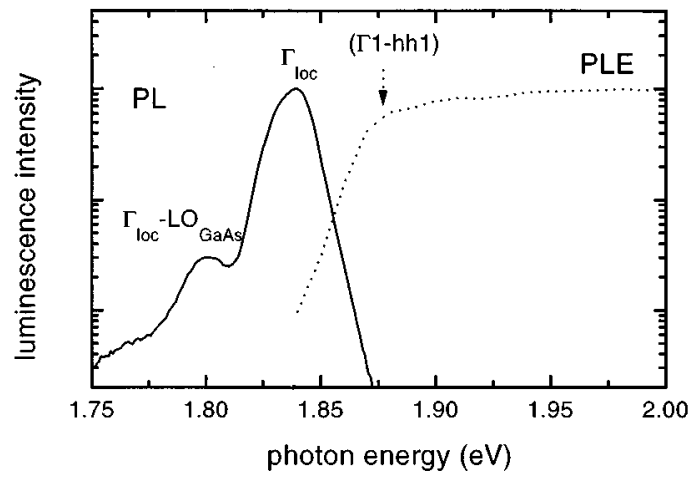

FIG. 1. PL (solid) and PLE (dotted) spectra of the sample at $5 \mathrm{~K}$ lattice temperature. The different transitions are labeled.

The observed shift between the PL and the PLE edge amounts to about twice the width of the PL. In strongly inhomogeneously broadened type-I samples, this shift is expected to be about $60 \%$ of the inhomogeneous linewidth ${ }^{21}$ at low temperatures. The observed larger shift in our sample is a consequence of the type-II band alignment, giving rise to an additional relaxation channel for the electrons via the $X_{z} 1$ states to deeper localized $\Gamma 1$ states. This relaxation mechanism is displayed schematically in Fig. 2. The $X_{z} 1$ states act only as intermediate states. This is due to their weak radiative recombination, which results in a backscattering into deeply localized $\Gamma 1$ states during the long type-II lifetime in spite of the low density of such type-I states. Consequently, the PL is of type-I, as is confirmed by the short PL decay time of about 200 ps, which is faster than for an indirect type-II transition. Taking these results together implies that the PL originates from localized ( $\Gamma 1-\mathrm{hh} 1)$ excitons, with an energy below the localized $\left(X_{z} 1-\mathrm{hh} 1\right)$ excitons in their neighborhood. In the following, we call these states local minima states $\Gamma_{\text {loc }}$.

The side peak around $1.80 \mathrm{eV}$ can accordingly be attrib-

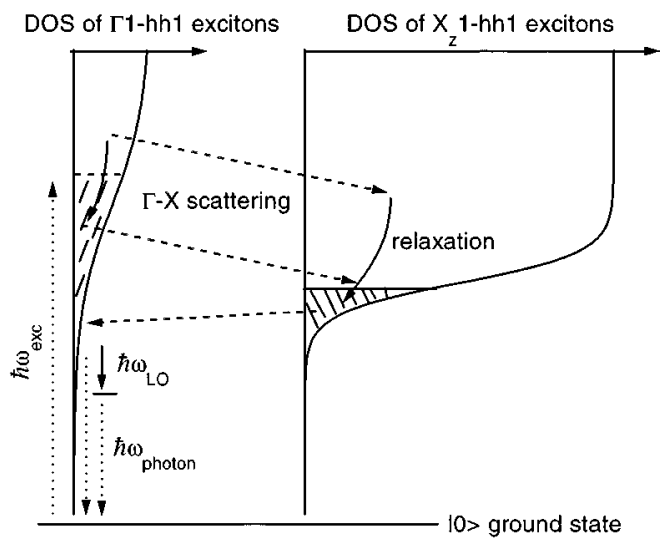

FIG. 2. Relaxation scheme of optically excited electron-hole pairs into ( $\Gamma 1-\mathrm{hh} 1)$ local minima exciton states. From these states, the excitons decay radiatively, either with or without the emission of an GaAs LO phonon, as indicated by the dotted arrows. The relaxation involves the $\left(X_{z} 1-\mathrm{hh} 1\right)$ excitons as transport states. The localized states at the band edges of the densities of states (DOS) are hatched. uted to a GaAs LO phonon replica of the PL from the localized $(\Gamma 1-h h 1)$ excitons, ${ }^{22}$ in agreement with the energy difference to the main peak of about $36 \mathrm{meV}$. The low-energy tail of the PLE shows an exponential behavior. It is due to the inhomogeneous broadening of the ( $\Gamma 1-\mathrm{hh} 1)$ transition, caused by layer thickness fluctuations. They give rise to an exponentially decreasing density of localized states below the band gap $D_{\Gamma}(E) \propto \exp \left(E / \epsilon_{\Gamma}\right)$ with a tailing parameter $\epsilon_{\Gamma}$ of $8 \mathrm{meV}$.

The high-energy side of the PL peak around $1.84 \mathrm{eV}$ shows an exponential decay with $4.6 \mathrm{meV}$ decay constant $\epsilon_{\mathrm{PL}}$. This is smaller than the tailing parameter $\epsilon_{\Gamma}$. The deviation can be explained by the relaxation via the $X_{z} 1$ states. The PL is due to local minima states. The density of such states at the energy $E$, which have to be below the ( $X_{z} 1$-hh1) excitons in their neighborhood, is determined by $D_{\Gamma}(E)$ and by the density of $\left(X_{z} 1-\mathrm{hh} 1\right)$ excitons below, given by $\left(\int_{-\infty}^{E} D_{X}(\epsilon) d \epsilon\right)^{-1} \propto \exp \left(E / \epsilon_{X}\right)$ with the tailing parameter $\epsilon_{X}$ of the $\left(X_{z} 1-\mathrm{hh} 1\right)$ transition. Due to the higher effective mass of the $X_{z} 1$ subband, $\epsilon_{X}$ is expected to be lower than $\epsilon_{\Gamma}$.

Accordingly, the PL intensity $I_{\mathrm{PL}}$ is proportional to $D_{\Gamma}(E)$ divided by the density of localized $\left(X_{z} 1-\mathrm{hh} 1\right)$ states below:

$$
I_{\mathrm{PL}} \propto \exp \left[E\left(\epsilon_{\Gamma}^{-1}-\epsilon_{X}^{-1}\right)\right] \propto \exp \left(E / \epsilon_{\mathrm{PL}}\right)
$$

Using $\epsilon_{\Gamma}$ and $\epsilon_{\mathrm{PL}}$ from the experiment, $\epsilon_{X}$ is calculated from this equation to be $2.9 \mathrm{meV}$. The ratio $\epsilon_{\Gamma} / \epsilon_{X}$ of about three is comparable to the inverse ratio of the excitonic reduced masses $\mu_{X} / \mu_{\Gamma}$ of approximately four, as expected from the fluctuation in quantization energies by thickness fluctuations.

\section{EXCITON RELAXATION}

In order to investigate the exciton relaxation within the localized type-I states, which are the origins of the PL, we measure the PL dynamics after pulsed resonant and nonresonant photoexcitation. Since the PL is well below the ( $\Gamma 1-\mathrm{hh} 1)$ band edge, such a relaxation is essentially only possible via an $\left(X_{z} 1\right.$-hh1) intermediate state. It involves the emission of acoustical phonons (phonon assisted

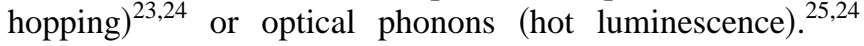
During the relaxation, the density of lower lying final states decreases. Consequently, the relaxation slows down and at some energy, the hopping time becomes longer than the radiative decay time. At this energy, the maximum of the steady state PL is expected. The involved localized states exhibit long dephasing times due to the reduced interaction with phonons. ${ }^{26}$ The localization of electrons and holes at different spatial positions, leading to a weak, long living $\mathrm{PL}^{27}$ has no significant influence on the results presented here.

The time-resolved PL of the sample after resonant excitation of low intensity, shown in Fig. 3, reveals two contributions. These are a resonant part, exhibiting the spectral shape of the exciting laser, and a nonresonant part, which is given by the density of localized ( $\Gamma 1-\mathrm{hh} 1)$ states and the exciton relaxation efficiency.

The PL spectra for different time delays [Fig. 3(a)] show 


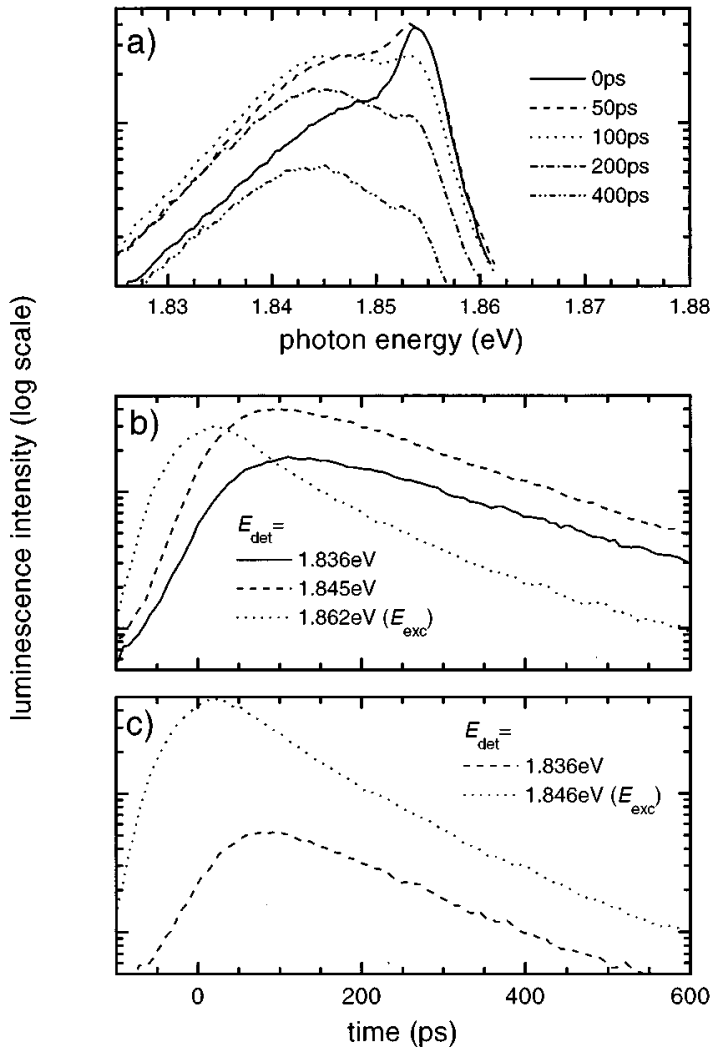

FIG. 3. Time-resolved PL of the sample after resonant excitation with $0.8 \mu \mathrm{J} / \mathrm{cm}^{2}$. (a) Spectra at various delay times for 1.854 $\mathrm{eV}$ excitation energy. PL time evolutions for the excitation photon energies $E_{\text {exc }}$ of $1.862 \mathrm{eV}$ (b) and $1.846 \mathrm{eV}$ (c) for resonant and nonresonant detection photon energies $E_{\text {det }}$, as labeled.

an increasing contribution of nonresonant PL with increasing delay time, a signature of the exciton relaxation into deeper localized states. During the first 100 ps, the relaxation leads to a change of the spectral shape without a major change in the integrated intensity. At longer times, the relaxation slows down and the integrated PL intensity decreases due to the recombination of the excitons. The resonant part is still observable at longer time delays, shifting slightly to lower energies.

The relaxation of the resonantly excited excitons can alternatively be seen in the time-resolved PL of the resonant and nonresonant part for different excitation energies, shown in Figs. 3(b),(c). The resonant PL shows a fast, initial decay due to the relaxation of a part of the resonant excitons, while the nonresonant PL shows a delayed maximum due to the in-scattering from the higher lying resonantly excited states.

After this initial fast relaxation, at delay times longer than the radiative lifetime of about 200 ps, both the resonant and nonresonant PL decay nearly with the same rate due to radiative recombination. This shows that the relaxation is, in addition to the transition energy, dependent on the microscopic arrangement of localized states in the vicinity of the resonant state. A state which has the lowest energy of all localized states within its extension (a local minimum state) is hindered from further relaxation by the vanishing overlap integral with lower states. The states which remain occupied after the fast relaxation thus are local minima. They cannot
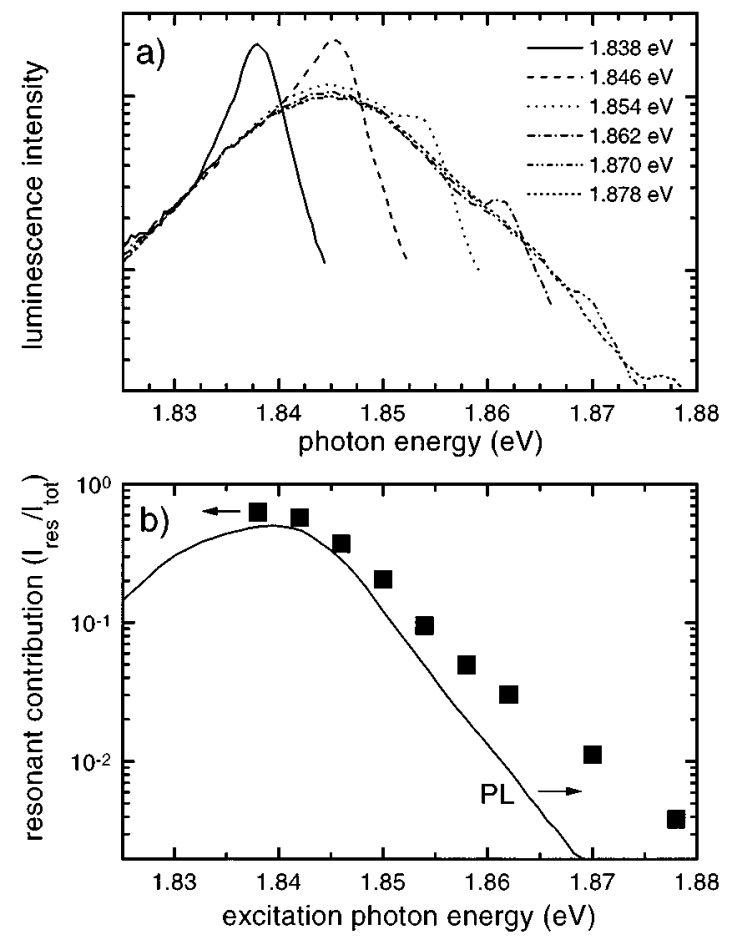

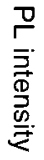

FIG. 4. (a) PL spectra after resonant excitation with 0.8 $\mu \mathrm{J} / \mathrm{cm}^{2}$ at various excitation energies at $240 \mathrm{ps}$ delay time, normalized to their low-energy wing. (b) Fraction of the resonant contribution to the PL at 240 ps delay time depending on the excitation photon energy. The time-integrated PL under off-resonant excitation is displayed for comparison.

be exclusively populated by direct optical excitation, but are selected by the exciton relaxation.

The PL spectra at 400 ps delay time, after the fast relaxation, are shown in Fig. 4(a) for various excitation energies. All spectra are normalized to their low-energy wing. This wing shows a constant shape for the different excitation energies and is thus mainly given by the density of the local minima.

In addition to the common low-energy tail, the PL of the nearly unrelaxed excitons is visible at the excitation energy. This resonant PL intensity $I_{\text {res }}$ increases with decreasing excitation energies, as displayed in Fig. 4(b). Here, the ratio between $I_{\text {res }}$ and the total PL intensity $I_{\text {tot }}$ at 400 ps delay time is displayed versus the excitation photon energy. Below the maximum of the nonresonant PL (solid line), essentially all PL is resonant. Consequently, there are mainly local minima states below this energy. Above, the ratio decreases exponentially, showing the smaller fraction of local minima states in the total density of states at higher energies. The decay to higher energies has a decay constant of $7.2 \mathrm{meV}$, which is between the tailing parameter $\epsilon_{\Gamma}$ of $8 \mathrm{meV}$ and the PL decay $\epsilon_{\mathrm{PL}}$ of $4.6 \mathrm{meV}$ (Fig. 1).

The shift of the PL maximum with a delay time after nonresonant excitation at $1.9 \mathrm{eV}$ is displayed in Fig. 5(a). We compare it with the shift expected for a hopping relaxation in a two-dimensional system, as it was proposed by Golub et al..$^{28}$

$$
E(t)=2 \epsilon_{\Gamma} \ln \left(\ln \left(\omega_{0} t\right)\right)+\text { const. }
$$



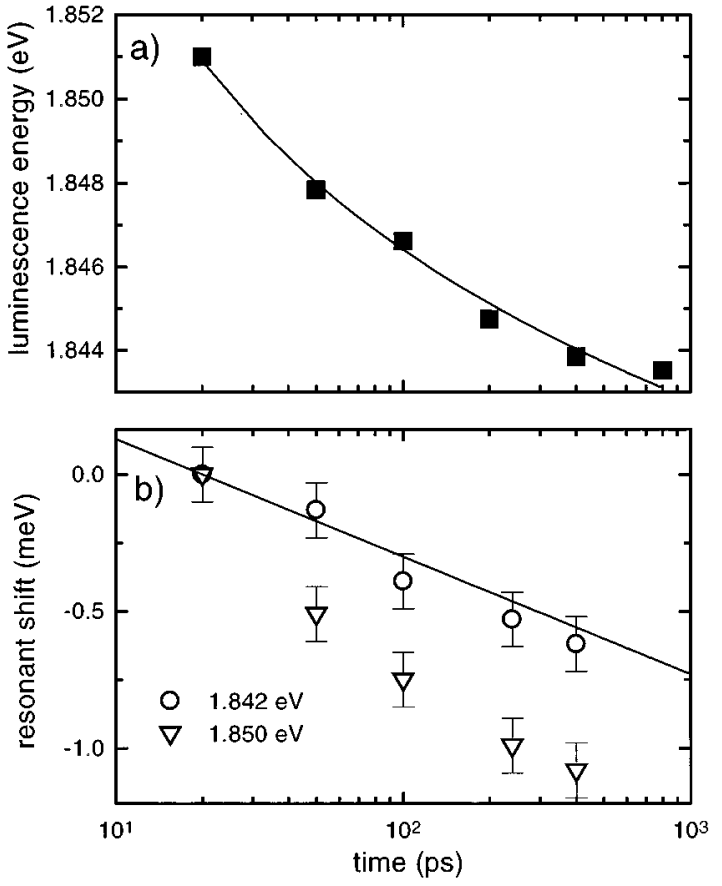

FIG. 5. (a) Shift of the PL maximum with delay time after off-resonant excitation at $1.9 \mathrm{eV}$. The curve is a fit by a hopping model using Eq. (2). (b) Shift of the resonant PL peak with delay time for two different excitation photon energies $1.842 \mathrm{eV}$ and $1.850 \mathrm{eV}$. The solid curve shows the shift expected for a multiple trapping process using Eq. (3).

In this model are assumed an exponential tail of localized states, with a tailing parameter $\epsilon_{\Gamma}$, and a hopping frequency of $\omega_{0}$ for fully overlapping states, independently of their energy difference. The tunneling probability between the states is modeled by an exponential decay with their spatial separation using a constant localization length. The exponential tail of the density of states in our sample is determined by the PLE (Fig. 1). The hopping between overlapping states is mediated by acoustic phonon emission. The curve in Fig. 5(a) is a fit to the experimental data with this model, giving the value $\omega_{0}=0.3 \mathrm{ps}^{-1}$. The extracted scattering time of 3.3 ps is within the expected range for acoustic phonon emission. ${ }^{29}$ This supports the validity of the applied model for the exciton relaxation in our sample.

The slight shift of the resonant part with time [Fig. 5(b)] was attributed previously ${ }^{25}$ to the multiple trapping relaxation within localized states. There is a difference between the shifts for excitation at the peak of the nonresonant PL $(1.842 \mathrm{eV})$, at which most of the excitons do not relax significantly, and for excitation on the high-energy wing of the nonresonant PL $(1.850 \mathrm{eV})$, at which most of the excitons relax by hopping during their lifetime. This difference can be explained by the increasing relaxation probability of the localized excitons with increasing energy as it is shown in Fig. 3(a). On the high-energy side of the resonant PL, a larger fraction of the resonantly excited excitons relaxes than on the low-energy side, leading to an apparent redshift of the resonant PL. By multiplying the initial resonant PL, which exhibits a width of $4 \mathrm{meV}$ from the exciting laser pulse, by this relaxation probability, we can determine the corresponding
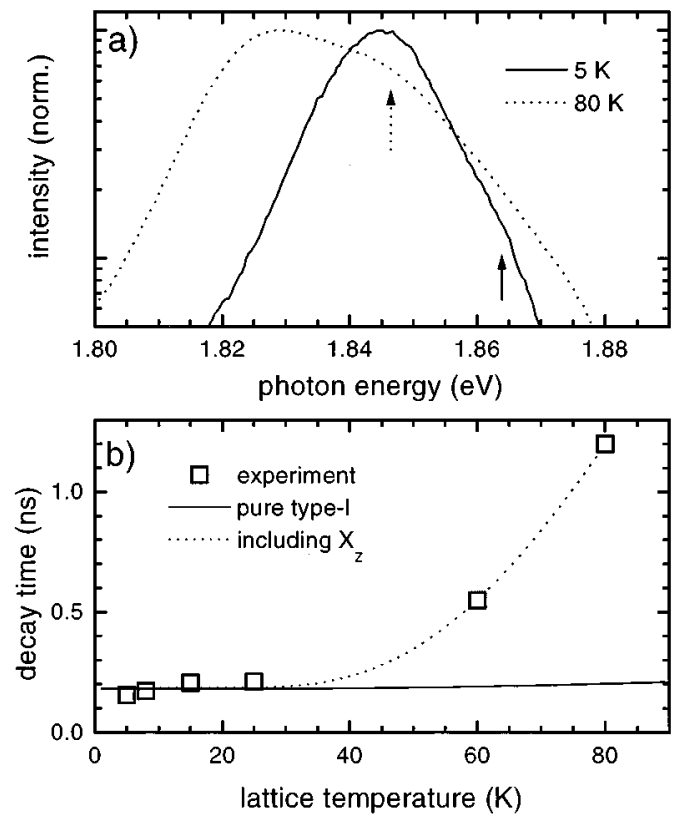

FIG. 6. Time-integrated PL spectra (a) and spectrally integrated PL decay time (b) for various lattice temperatures. The dotted curve in (b) is a fit using Eq. (5), the solid curve is expected for a pure type-I structure using Eq. (4).

redshift to $0.5 \mathrm{meV}$, which matches the difference for the two excitation energies [Fig. 5(c)]. The remaining shift common for both excitation energies shows a logarithmic dependence with delay time. Such a logarithmic shift

$$
\Delta E=k_{B} T \ln \left(\nu_{0} t\right)
$$

is expected for a multiple trapping process. ${ }^{25}$ The expected time dependence is displayed in Fig. 5(b) for a lattice temperature $T$ of $5 \mathrm{~K}$ and a $\nu_{0}$ of $20 \mathrm{ps}$, fitting well the experimental data. The multiple trapping requires an activation of the excitons above the mobility edge, which is given in our sample by the extended $\left(X_{z} 1\right.$-hh1) exciton states. The difference between the scattering frequencies $\nu_{0}$ and $\omega_{0}$ might be due to the thermal activation needed for the multiple trapping. However, the attribution to the multiple trapping process is not unequivocal, ${ }^{24}$ since the difference to the double logarithmic hopping relaxation [Eq. (2)] is not resolved by the experimental accuracy.

\section{EXCITONIC LIFETIME: INFLUENCE OF THE TYPE-II TRANSITION}

Let us discuss now the influence of the $X_{z} 1$ subband on the temperature and density dependent exciton dynamics. Both by increasing the lattice temperature or the carrier density, the higher lying ( $\left.X_{z} 1-\mathrm{hh} 1\right)$ states (Fig. 2) become occupied, leading to an increase of the PL decay time. Figure 6 shows the time-integrated PL spectra at $5 \mathrm{~K}$ and $80 \mathrm{~K}$ together with the temperature dependence of the spectrally integrated PL decay time. The PL spectrum at $80 \mathrm{~K}$ shows the temperature induced redshift of the band gap and a broadening of the high-energy wing. The PL decay time $\tau$ shows a 
strong, nonlinear increase above $40 \mathrm{~K}$. This is different from the temperature dependence of the PL decay time of excitons in type-I quantum wells, ${ }^{30}$ which is given by the fraction of excitons in the radiative states at the excitonic band edge:

$$
\tau \propto \frac{\Delta(T)}{1-\exp \left(-\frac{\Delta(T)}{k_{B} T}\right)} .
$$

Here, $\Delta(T)$ denotes the energy width of the radiative exciton states band. For most quantum wells, this radiative zone is larger than the width expected from the homogeneous width of the excitons or from the dispersion of the exciton within the light cone. This is due to interface fluctuations, leading to an inhomogeneous broadening of the transition, and thus allowing for a larger momentum-space region of excitons to couple to the photons. In this sense, the excitons in quantum wells are in most real samples localized at the band edge.

In our case, the width of the radiative zone $\Delta(T)$ would be at least the PL linewidth of $18 \mathrm{meV}$. For this value, Eq. (4) predicts a nearly constant decay time up to $80 \mathrm{~K}$ [solid line in Fig. 6(b)], in contrast to the experimental data.

Consequently, to explain the observed strong increase of the decay time, there must exist other dark exciton states besides the type-I excitons outside the radiative zone. These states are the $\left(X_{z} 1\right.$-hh1 $)$ states, which exhibit a long radiative lifetime. The density of localized ( $\Gamma 1-\mathrm{hh} 1)$ states emitting the PL is only about $2 \%$ of the ( $\Gamma 1$-hh 1$)$ density of states, as we estimate by the comparison of the PLE signal (Fig. 1) at the band edge of the ( $\Gamma 1-\mathrm{hh} 1)$ transition with the PLE signal at the energy of the PL maximum. The $\left(X_{z} 1-\mathrm{hh} 1\right)$ transition starts slightly above the energy of these localized $\Gamma 1$ states, and its density of states is about four times larger than that of the (Г1-hh1) transition due to its larger effective mass.

To model the influence of both exciton bands, we distribute the excitons thermally over a small, steplike density $D_{\Gamma}$ of radiative states with the decay time $\tau_{0}$, representing the localized ( $\Gamma 1$-hh1) states, and a large, steplike density $D_{X}$ of dark states, starting the energy $\Delta E$ above $D_{\Gamma}$ and representing the $\left(X_{z} 1-\mathrm{hh} 1\right)$ states. Hereby, we have assumed a thermal energy less than the inhomogeneous broadening of the type-I transition, which is fulfilled within the considered temperature range. The fraction of excitons in the radiative states $D_{\Gamma}$ give the temperature dependent decay time $\tau$, which accordingly reads

$$
\tau=\tau_{0}\left[1+\frac{D_{X}}{D_{\Gamma}} \exp \left(-\frac{\Delta E}{k_{B} T}\right)\right] .
$$

The value $D_{X} / D_{\Gamma}$ is expected to be about 200 from the previous considerations. The experimental data are described well by this dependence [Fig. 6(b)] using $\Delta E=21 \mathrm{meV}$, $\tau_{0}=185 \mathrm{ps}$ and $D_{X} / D_{\Gamma}=120$. The energy splitting $\Delta E$ is also visible by the kink in the PL spectrum, marked by an arrow in Fig. 6(a).

The function of the $X_{z} 1$ subband as a carrier reservoir is also important at high exciton densities. This is demonstrated in the time-resolved PL after high-intensity excitation,

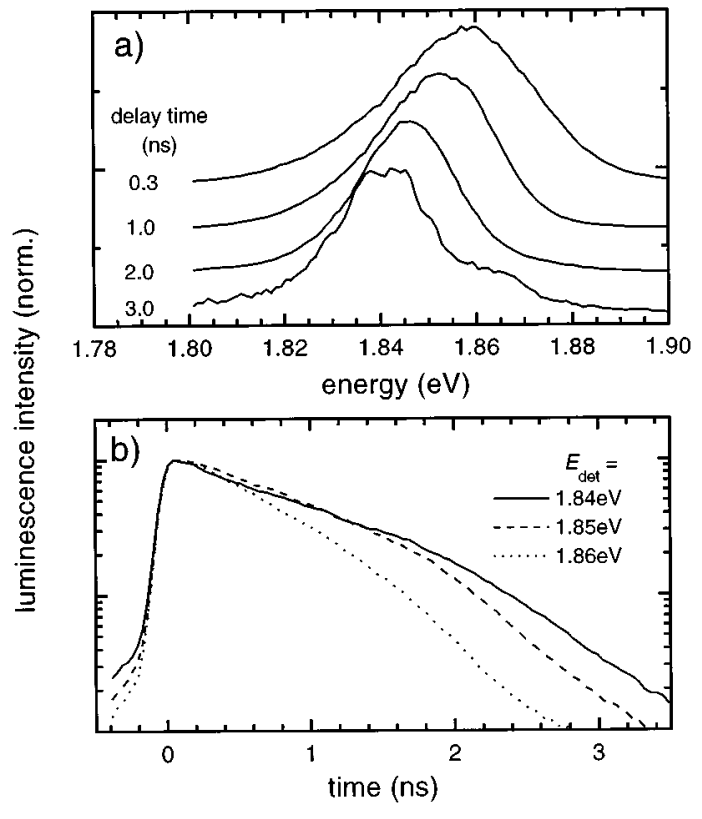

FIG. 7. PL of the sample after high optical excitation with 50 $\mu \mathrm{J} / \mathrm{cm}^{2}$ at $2.46 \mathrm{eV}$ and a lattice temperature of $4.5 \mathrm{~K}$. (a) Spectra for various delay times, as indicated. The curves are vertically offset for clarity. (b) Time-resolved PL for different detection photon energies, as indicated.

shown in Fig. 7. The excitation intensity of $50 \mu \mathrm{J} / \mathrm{cm}^{2}$ amounts to a pair density of about $3 \times 10^{12} / \mathrm{cm}^{2}$. The PL spectra for different delay times [Fig. 7(a)] reflect the decrease of the carrier density and temperature. At long times, the energy splitting of about $20 \mathrm{meV}$ between the peak of the localized type-I PL and the onset of the type-II states becomes visible by a shoulder on the high-energy side. This is a consequence of the carrier relaxation, as depicted in Fig. 2 . The temporal evolution of the integrated PL intensity shows a peculiar behavior [Fig. 7(b)]. The initial decay time of $1 \mathrm{~ns}$ decreases around $1.5 \mathrm{~ns}$ delay time to $0.5 \mathrm{~ns}$. These dynamics are opposite to the slowing down of the PL decay in an inhomogeneous, decoupled system with different decay times. ${ }^{11}$ We attribute the increased initial decay time to the influence of the $X_{z} 1$ electron reservoir. Shortly after excitation, the $X_{z} 1$ and the $\Gamma 1$ are filled up to the quasi-Fermi level above the localized states of the $X_{z} 1$ subband. Hence, the carriers are mobile and the electron distribution is in quasiequilibrium. Due to recombination, the density drops with time and the quasi-Fermi level shifts below the $X_{z} 1$ subband. At this point, the localized $\Gamma 1$ states are no longer refilled by the carriers from the $X_{z} 1$ states and the decay time becomes faster.

\section{SUMMARY}

In summary, we have investigated the PL dynamics in a type-II GaAs/AlAs superlattice near the type-I to type-II crossover. We have demonstrated that the dominant PL contribution is due to localized states of the type-I transition. The carrier relaxation within these localized states by 
phonon-assisted hopping is found to be important for energies above the PL maximum. The relaxation dynamics can be described by a hopping model. ${ }^{28}$ The $X_{z} 1$ subband is found to increase the PL decay time for higher temperatures and carrier densities, due to its function as a carrier reservoir.

\section{ACKNOWLEDGMENTS}

We want to acknowledge R. Nötzel, H. P. Schönherr, and K. Ploog for the sample growth. This work was supported by the Danish Natural Science Research Council.
*Present address: Mikroelektronik Centret, DTU, Bldg. 345 east, DK-2800 Lyngby, Denmark. Electronic address: Langbein@mic.dtu.dk

${ }^{1}$ G. Danan et al., Phys. Rev. B 35, 6207 (1987).

${ }^{2}$ G. R. Olbright et al., Phys. Rev. B 44, 3043 (1991).

${ }^{3}$ H. van Kesteren et al., Phys. Rev. B 39, 13426 (1989).

${ }^{4}$ P. Lefebvre, B. Gil, H. Mathieu, and R. Planel, Phys. Rev. B 39, 5550 (1989).

${ }^{5}$ K. J. Moore, P. Dawson, and C. T. Foxon, Phys. Rev. B 38, 3368 (1988).

${ }^{6}$ M. Nakayama et al., Phys. Rev. B 49, 13564 (1994).

${ }^{7}$ V. Voliotis et al., Phys. Rev. B 49, 2576 (1994).

${ }^{8}$ T. Ando, Phys. Rev. B 47, 9621 (1993).

${ }^{9}$ Y. Fu, M. Willander, E. L. Ivchenko, and A. A. Kislev, Phys. Rev. B 47, 13498 (1994).

${ }^{10}$ G. D. Gilliland et al., Phys. Rev. Lett. 71, 3717 (1993).

${ }^{11}$ J. F. Angell and M. D. Sturge, Phys. Rev. B 48, 4650 (1993).

${ }^{12}$ L. P. Fu et al., Phys. Rev. B 52, 2682 (1995).

${ }^{13}$ J. F. Heffernan, J. Hegarty, and R. Planel, Phys. Rev. B 52, 7818 (1995).

${ }^{14}$ A. B. Dzyubenko and G. E. W. Bauer, Phys. Rev. B 51, 14524 (1995).

${ }^{15}$ D. Gammon, B. V. Shanabrook, and D. S. Katzer, Phys. Rev.
Lett. 67, 1547 (1991)

${ }^{16}$ A. Ourmazd, D. W. Taylor, J. Cunningham, and C. W. Tu, Phys. Rev. Lett. 62, 933 (1989).

${ }^{17}$ C. A. Warwick and R. F. Kopf, Appl. Phys. Lett. 60, 386 (1992).

${ }^{18}$ M. F. Pereira, I. Galbraith, S. W. Koch, and G. Duggan, Phys. Rev. B 42, 7084 (1990).

${ }^{19}$ M. M. Dignam and J. E. Sipe, Phys. Rev. B 41, 2865 (1990).

${ }^{20}$ K. Fujiwara et al., Phys. Rev. B 49, 1809 (1994).

${ }^{21}$ F. Yang, M. Wilkinson, E. J. Austin, and K. P. O'Donnel, Phys. Rev. Lett. 70, 323 (1993).

${ }^{22}$ K. J. Nash, M. S. Skolnick, P. A. Claxton, and J. S. Roberts, Phys. Rev. B 39, 5558 (1989).

${ }^{23}$ M. Zachau, J. A. Kash, and W. T. Masselink, Phys. Rev. B 44, 8403 (1991).

${ }^{24}$ H. Kalt et al., Phys. Rev. B 45, 4253 (1992).

${ }^{25}$ J. H. Collet et al., Phys. Rev. B 43, 6843 (1991).

${ }^{26}$ H. Schwab, V. G. Lyssenko, and J. M. Hvam, Phys. Rev. B 44, 3413 (1991).

${ }^{27}$ A. Frommer et al., Phys. Rev. B 49, 2935 (1994).

${ }^{28}$ L. E. Golub, E. L. Ivchenko, and A. A. Kiselev, J. Opt. Soc. Am. B 13, 1199 (1996).

${ }^{29}$ T. Takagahara, Phys. Rev. B 31, 6552 (1985).

${ }^{30}$ J. Feldmann et al., Phys. Rev. Lett. 59, 2337 (1987). 